\title{
Shock Wave Pressure Analysis of Underground Structure Associated with Buried Natural-gas Pipeline Explosion
}

\author{
Zhongxin ZHOU \\ State Key Laboratory for Disaster Prevention \& Mitigation \\ of Explosion \& Impact, \\ PLA University of Science and Technology \\ Nanjing, China \\ Hualin FAN \\ State Key Laboratory of Mechanics and Control of \\ Mechanical Structures \\ Nanjing University of Aeronautics and Astronautics \\ Nanjing, China \\ e-mail: fh102@mails.tsinghua.edu.cn
}

\author{
Jiannan ZHOU \\ State Key Laboratory for Disaster Prevention \& Mitigation \\ of Explosion \& Impact \\ PLA University of Science and Technology \\ Nanjing, China \\ e-mail: zjn_0414@163.com
}

Fengnian JIN

State Key Laboratory for Disaster Prevention \& Mitigation of Explosion \& Impact

PLA University of Science and Technology

Nanjing, China

e-mail: jinfn2009@163.com

Yin XU

State Key Laboratory for Disaster Prevention \& Mitigation of Explosion \& Impact PLA University of Science and Technology

Nanjing, China

\begin{abstract}
The full-bore rupture of a buried high-pressure natural-gas pipeline can lead to detonation waves that can pose a significant threat to underground structure in the vicinity of failure location. The dominant hazards are shock wave propagation through the soil around the underground structure from buried pipeline physical explosion. In this paper, a methodology is presented to evaluate hazard areas of underground structure relating the diameter, the operating pressure, the length of pipeline, soil characteristics, the distance between buried pipeline and underground structure. An effective thermodynamic method is proposed to estimate the energy of buried pipeline physical explosion. TNT equivalent model is utilized to convert buried pipelines physical explosion energy, two simplified models are proposed, concentrated mass and linear distribution per unit length, to comprehensively describe the effective physical explosion range of the longitudinal length of the pipeline. The methodology can be readily used by practicing engineers to calculate the shock wave pressure of existing or constructing underground structure subjected to pipeline physical explosion.
\end{abstract}

Keywords-buried gas pipeline; full-bore rupture; physical explosion; shock wave; TNT equivalence method

\section{INTRODUCTION}

It is often required to orthogonally cross subway lines due to the increasing market demand of city metro construction beneath the main gas pipeline routes due to the increasing market demand of city metro construction. Constructing new subway routes at a certain distance from existing buried pipeline requires the evaluation of possible pipeline full-bore rupture damage to the subway structures.

The importance of this kind of analysis is due to the fact that internal gas pipeline high-pressure generates a hoop tensile stress usually in the vicinity of yield. Thus, an external mechanical interference by third party, earthquake, or blast overpressure may cause pipeline full-bore rupture. Following the research, British Gas concluded that leaks may propagate to full-bore rupture if the ratio of operating stress to yield stress of a pipeline is greater than about 0.3 [1]. The full-bore rupture of a buried high-pressure natural-gas pipeline can lead to shock wave propagation filtering through the soil that can pose a significant threat to underground structures. The European Gas Pipeline Incident Data Group presents an overall failure rate of 0.575 per $1000 \mathrm{~km} \bullet y e a r$ based on the experiences over $1.47 \times 10^{6} \mathrm{~km} \cdot$ years of onshore natural gas-pipeline [2]. While there is an overall failure rate of 3 per $1000 \mathrm{~km} \cdot y e a r$ based on the experiences of natural gas and oil pipeline in China [3].

\section{MODELING}

Hazardous event of the credible worst case is the fullbore rupture of a high-pressure pipeline with physic explosion resulting shock wave. In this work, the proposed methodology is consisted of three parts: 1) an effective thermodynamic method for estimating physical explosion of the compressed gas energy, 2) The critical depth effect of buried pipeline explosion, 3) TNT equivalent model. 


\section{A. Effective Thermodynamic Method}

Thermodynamic effectiveness of the method is Crowl [4] application of the overall effectiveness of the thermodynamic method, based on isothermal expansion method, give analysis to calculate the largest container-constrained gas explosion energy equation, the expression is:

$$
E_{\text {Brode }}=P_{2} V\left[\ln \left(\frac{P_{2}}{P_{0}}\right)-\left(1-\frac{P_{0}}{P_{2}}\right)\right]
$$

\section{B. Effect of Depth}

Explosions beneath the ground surface produce a radially expanding shock wave propagating through the soil. In the analyses, a ground shock coupling factor is employed to quantify the enhancement of the compressive shock wave as a function of the higher confinement provided by a higher depth. A relationship between depth of burst, measured at the center of the charge, and charge size is shown in Figure 1 for different values of the coupling factor. For combinations of charge weight and depth plotting below the $f=1$ curve, peak velocity and pressure histories have to be reduced using the appropriate coupling factor.

\section{TNT Equivalent Model}

The scope of buried pipeline explosion applied on the subway shield structure is determined; employ the effectiveness of thermodynamics method to calculate. Aiming at the buried gas pipeline occur complete physical explosion. Take $1 \mathrm{~m}$ per unit length of the gas pipeline in the calculation process to calculate.

Use of TNT equivalent method to convert, $W_{T N T}$ is equivalent quality of TNT:

$$
W_{T N T}=m_{P} P_{2} \frac{\pi \times d^{2}}{4} \times L\left[\ln \left(\frac{P_{2}}{P_{0}}\right)-\left(1-\frac{P_{0}}{P_{2}}\right)\right] / Q_{T N T}
$$

Physical explosion beneath the underground surface produce a radically expanding shock wave through the soil. The relationships between exponential pressure and time are given in Protective Design Manuals, such as TM5-855-1 [5].

\section{SimPlified ANALYSIS AND DISCUSSION}

When the pipeline explodes, the explosive energy transforms into a small part of the kinetic energy of fragments and most of the energy of the stress wave. According to the TNT equivalent method to convert, buried pipeline explosion can be seen as a linear strip TNT explosion source; two simplified models are proposed, concentrated mass and linear distribution per unit length, to comprehensively describe the effective physical explosion range of the longitudinal length of the pipeline.

\section{A. Concentrated Mass Model}

Continuous pipeline is simplified. It will simplify the unit length of pipe into the middle of a little concentrated mass equivalent TNT charge block. Points from the pipe directly above the center point distance A (marked in the diagram, is shown in Figure 2) is $0.5,1.5,2.5,3.5 \ldots, \mathrm{n}-0.5$.

\section{B. Linear Distribution Model}

The buried pipe is simplified as a continuous strip charge calculation. For TNT, specific density $\rho_{T N T}=1630 \mathrm{~kg} / \mathrm{m}^{3}[6]$, when $L=1 \mathrm{~m}$, as defined stripped charge sectional area of explosives.

\section{Analysis and Discussion of Examples}

As it can be seen from Figure 2, a shield tunneling, the outer diameter of shield tunnel is $6.2 \mathrm{~m}$. Shield zone under certain mileage orthogonally under-cross the China WestEast Gas Pipeline, piping and line angle are approximately $80^{\circ}$, West-East Gas Pipeline is $\Phi 1016 \mathrm{~mm}$ diameter steel pipe (wall thickness $25 \mathrm{~mm}$ ), pressure $10 \mathrm{MPa}$, Tube top depth is about $5.5 \mathrm{~m}$, the minimum vertical distance is about $13.4 \mathrm{~m}$ between shield and pipeline. Soil properties of the medium parameters can be seen in the table 1 .

1) The effective length of the physical pipeline explosion:

In the project example, Critical depth pipeline explosion crater is $4.014 \mathrm{~m}$; depth of the pipe is $5.5 \mathrm{~m}$. Pipeline depth is greater than critical depth; around the tube formation of explosive gas pressure is within the chamber and the static pressure of the soil balance [7]. Explosion pipeline calculates the sum points of the peak compression pressure values.

As can be seen from Figure 3 and 4, two simplified model calculation results are consistent; Table 2, when the pipelines explode in $20 \mathrm{~m}$ length, the difference between the two is less than $3 \%$. In theory, continuous charge is more reasonable assumption, but for engineering applications, simplified as point source charge is more convenient in the calculation. From Figure 3 and 4, it can be seen with the increase in the length of the pipeline, the wave pipeline explosion peak overpressure is gradually stability. Therefore, it is recommended to take physical gas pipeline explosion effective length of $40 \mathrm{~m}$.

2) The influence of different pipeline depth on underground structural damage:

The vertical distance is discussed between the shield away from the pipeline, gas pipeline length is $40 \mathrm{~m}$, and the peak stress value changes in the relationship between the vertical distances. As can be seen from the Figure 5, the distance between buried gas pipeline and the shield is greater, the stress wave peak attenuate sooner, the stress applied on the subway shield is smaller, more security the metro shield is. Within $1 \mathrm{~m}-2 \mathrm{~m}$ range, peak stress wave attenuate rapidly, attenuation factor is about 6 times, when the distance is more than $2 \mathrm{~m}$, attenuated rate decreases; gradually stabilize after $4 \mathrm{~m}$ range. As can be seen Table 3, under the $2 \mathrm{~m}$ and $12 \mathrm{~m}$ depth, the stress wave peak ratio is about 90 times. Therefore, the gas pipeline should be buried in keeping a certain safe distance from the metro shield to make a small impact on physical explosion shield.

3) The influence of different pipeline pressure on underground structural damage:

The vertical distance is $4 \mathrm{~m}, 6 \mathrm{~m}, 8 \mathrm{~m}$ from the Metro to the pipeline shield, the length of the gas pipeline takes 
effective length of $40 \mathrm{~m}$, the relationship between the inner tube gas pressure and stress wave peak is investigated. Pipe pressure has a great impact on the stress wave, as can be seen from Figure 6, with the pressure increasing, stress wave peak overpressure also increased, when the pressure inside the pipe is large, approximately they are proportional. As can be seen from Figure 6, the overpressure effects of the distance between the pipe and the shield are more obvious than the overpressure inside the tube.

\section{CONCLUSIONS}

According to the research, it is concluded that:

(1) Using equivalent TNT model and converting equivalent TNT of underground pipelines physical explosion energy, we proposed two calculation models: Simplified concentrated mass per unit length and to linear simplified. Use soil free field compression wave to calculate peak formula, calculate the soil compression wave peak of Metro Shield highest point A, and confirm the consistency of the equivalent TNT charge concentration mass and linear simplified model, recommend to take in engineering applications linear simplified calculate and analyze.

(2) Taking the example of underground pipeline project of a subway shield, by using empirical formula of free field soil compression wave, give the total variation of the peak value of the compression wave at the highest point of the subway structure. It is suggested that the effective range of the longitudinal length of the pipeline explosion is $40 \mathrm{~m}$, and the peak variation of the free field at the highest point of the shield tunnel under different distance conditions are studied, obtain the effective influence range of underground gas pipeline physical explosion to shield tunnel. The closer is the distance; the greater is the peak free-field stress. Within 1m$2 \mathrm{~m}$ range, peak stress wave attenuates rapidly, attenuation factor is about 6 times, there is a turning point at $2 \mathrm{~m}$, the attenuated rate decreases, gradually stabilize after $4 \mathrm{~m}$. Compare $2 \mathrm{~m}$ with $12 \mathrm{~m}$, the stress wave peak ratio is about 90 times. Therefore, the gas pipeline should be buried at a certain distance from metro shield.

(3) Pipe pressure has a greater impact on the underground structure, with the inner tube pressure increase, the stress wave peak overpressure also increase, when the pressure inside the pipe is large, both of them are approximately proportional.

TABLE I. The Nature OF THE SOIL Medium Parameters

\begin{tabular}{|c|c|c|c|}
\hline $\begin{array}{c}\text { Soil } \\
\text { No. }\end{array}$ & $\begin{array}{c}\text { Soil } \\
\text { name }\end{array}$ & $\begin{array}{c}\text { Layer bottom } \\
\text { elevation }(\boldsymbol{m})\end{array}$ & $\begin{array}{c}\text { Thickness }(\boldsymbol{m}) \text { The } \\
\text { range of values } \\
\text { /average }\end{array}$ \\
\hline 1 & Backfill & $4.08 \sim-2.68 / 2.01$ & $0.3 \sim 6.80 / 2.67$ \\
\hline 2 & $\begin{array}{c}\text { Mucky } \\
\text { silty } \\
\text { clay }\end{array}$ & $0.4 \sim-15.28 /-2.69$ & $1.50 \sim 12.60 / 4.14$ \\
\hline 3 & $\begin{array}{c}\text { Silty } \\
\text { clay }\end{array}$ & $-3.46 \sim-7.16 /-4.78$ & $2.40 \sim 4.30 / 3.02$ \\
\hline
\end{tabular}

TABLE II. RELATIONSHIP OF 40M LONG PIPELINE STRESS WAVE Peak and the Vertical Distance From Gas PiPelines to Shield

\begin{tabular}{|c|c|c|c|c|c|c|c|c|}
\hline $\begin{array}{c}\text { Vertical } \\
\text { distance }\end{array}$ & $\mathbf{1}$ & $\mathbf{2}$ & $\mathbf{4}$ & $\mathbf{6}$ & $\boldsymbol{8}$ & $\mathbf{1 0}$ & $\mathbf{1 2}$ & $\mathbf{1 3}$ \\
\hline $\begin{array}{c}\text { Peak } \\
\text { overpressure }\end{array}$ & 6.06 & 1.07 & 0.19 & 0.07 & 0.03 & 0.02 & 0.01 & 0.01 \\
\hline
\end{tabular}

TABle III. The Relations BetweEn PiPEs PREsSURe With OVERPRESSURE VALUE

\begin{tabular}{|c|c|c|c|c|}
\hline \multirow{2}{*}{$\begin{array}{c}\text { Over } \\
\text { pressure }\end{array}$} & \multirow{2}{*}{$\begin{array}{c}\text { TNT } \\
\text { equivalent } \\
\text { quality }\end{array}$} & $\begin{array}{c}\mid c \\
\text { distance } \\
\text { of } \mathbf{4 m}\end{array}$ & $\begin{array}{c}\text { distance of } \\
\mathbf{6 m}\end{array}$ & $\begin{array}{c}\text { distance of } \\
\mathbf{8 m}\end{array}$ \\
\hline 3 & 1.470 & 0.0258 & 0.0093 & 0.0045 \\
\hline 4 & 2.258 & 0.0426 & 0.0154 & 0.0074 \\
\hline 5 & 3.110 & 0.0619 & 0.0224 & 0.0108 \\
\hline 6 & 4.030 & 0.0838 & 0.0302 & 0.0146 \\
\hline 7 & 4.989 & 0.1075 & 0.0388 & 0.0187 \\
\hline 8 & 5.989 & 0.1330 & 0.0480 & 0.0232 \\
\hline 9 & 7.023 & 0.1601 & 0.0578 & 0.0279 \\
\hline 10 & 8.087 & 0.1889 & 0.0682 & 0.0329 \\
\hline 11 & 9.179 & 0.2189 & 0.0790 & 0.0381 \\
\hline 12 & 10.296 & 0.2503 & 0.0903 & 0.0436 \\
\hline
\end{tabular}

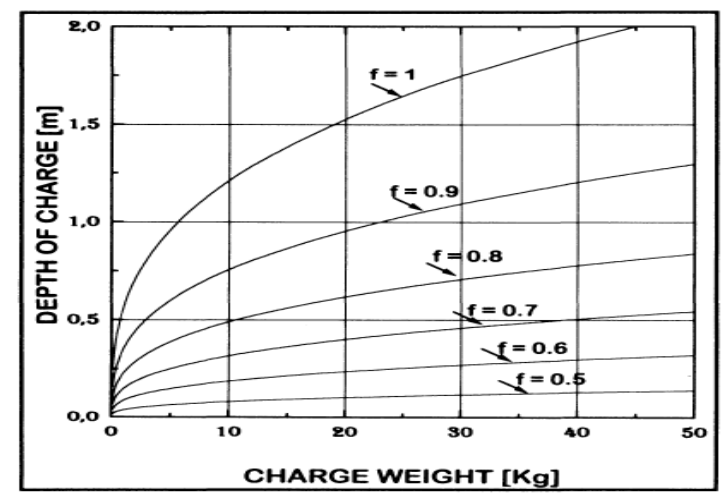

Figure 1. Ground blast coupling factor as a function of depth of burst and charge weight



Figure 2. A profile map of a gas pipeline and underground sturcture 


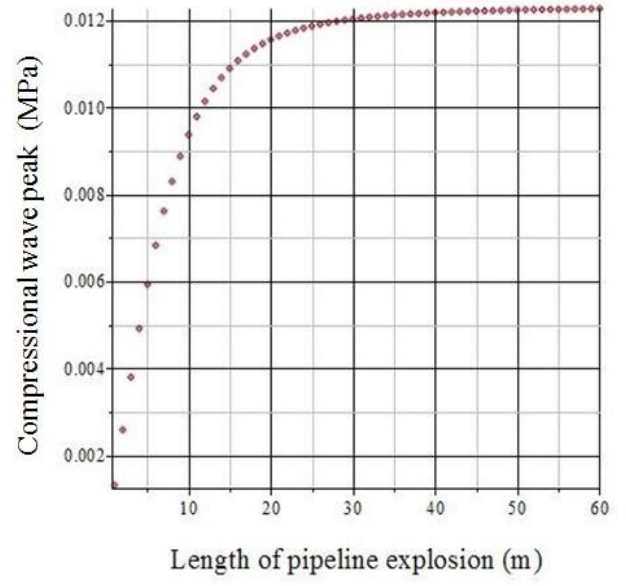

Figure 3. The relationship of the lumped explosive compression wave

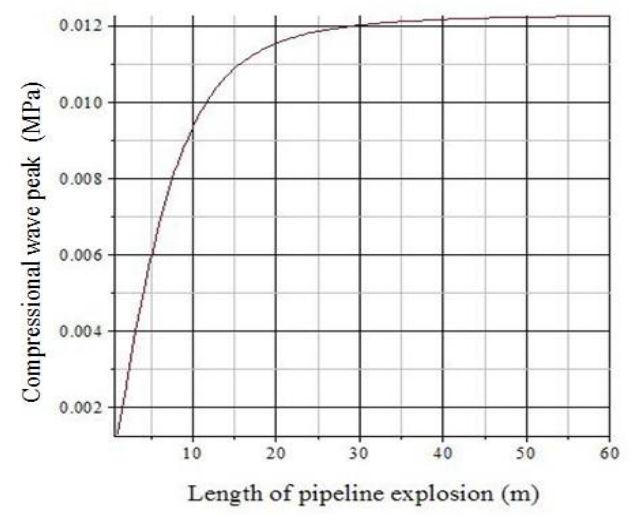

Figure 4. The relationship of the linear unit and length charge and compression wave



Figure 5. Relationship of compression wave crest value and the vertical distance between gas pipeline and shield

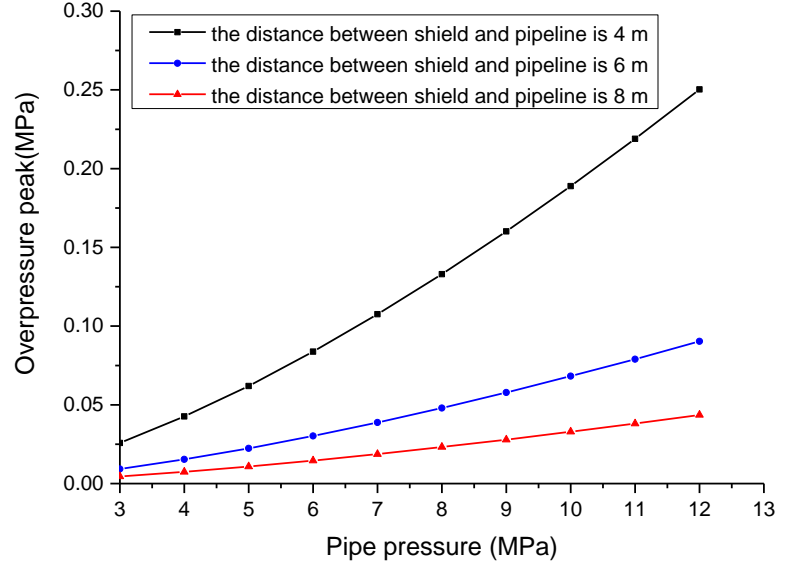

Figure 6 . The relations between pipes pressure with overpressure value

\section{ACKNOWLEDGMENT}

Supports from Natural Science Foundations of Jiangsu Province (BK20151448), National Natural Science Foundation of China (51308544, 51478465, and 51508567) are gratefully acknowledged.

\section{REFERENCES}

[1] Townsend, N.A., \& Fearnehough, G.D. (1986). Control of risk from UK gas transmission pipelines, Presented at the 7th Symposium on Line Pipe Research, AGA, Houston, October.

[2] Morgan, B. (1995). The importance of realistic representation of design features in the risk assessment of high-pressure gas pipeline, 5th International Conference and Exhibition Pipeline Reliability, Houston, Texas, September.

[3] MA Xiang-nan, DING hui. Research on Urban Buried Gas pipeline leak emergency resource scheduling [D]. Capital University of Economics and Business, 2010, 6.

[4] D.A.Crowl.Caculation the Energy of Explosion Using Thermodynamic Availability [J]. Journal of Loss Prevention in Process Industries, 1992, 5(2):109-118.

[5] TM5-855-1.foundamentals of protective design for conventional weapons. Vicksburg: US Army Engineers Waterways Experimental Station, 1986:1-333.

[6] Wang Yuanhui. Safety System Engineering [M]. Tianjin University Press, 1999.10

[7] Gun Wenbin, Ye Xushuang. The kinetic analysis of spherical Charge semi-infinite soil medium explosion [J] blasting principle, 1999,5 (1): $5-10$. 